\title{
Tritium labelling of a cholesterol amphiphile designed for cell membrane anchoring of proteins
}

\author{
Balázs Schäfer, ${ }^{a}$ Erika Orbán, ${ }^{b \dagger}$ Zoltán Kele, ${ }^{c}$ and Csaba Tömböly ${ }^{a *}$
}

Cell membrane association of proteins can be achieved by the addition of lipid moieties to the polypeptide chain, and such lipid-modified proteins have important biological functions. A class of cell surface proteins contains a complex glycosylphosphatidylinositol (GPI) glycolipid at the C-terminus, and they are accumulated in cholesterol-rich membrane microdomains, that is, lipid rafts. Semisynthetic lipoproteins prepared from recombinant proteins and designed lipids are valuable probes and model systems of the membrane-associated proteins. Because GPI-anchored proteins can be reinserted into the cell membrane with the retention of the biological function, they are appropriate candidates for preparing models

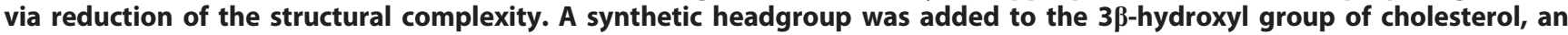
essential lipid component of rafts, and the resulting cholesterol derivative was used as a simplified GPI mimetic. In order to quantitate the membrane integrated GPI mimetic after the exogenous addition to live cells, a tritium labelled cholesterol anchor was prepared. The radioactive label was introduced into the headgroup, and the radiolabelled GPI mimetic anchor was obtained with a specific activity of $1.37 \mathrm{TBq} / \mathrm{mmol}$. The headgroup labelled cholesterol derivative was applied to demonstrate the sensitive detection of the cell membrane association of the anchor under in vivo conditions.

Keywords: tritium; cholesterol; membrane anchor; lipoprotein

\section{Introduction}

A unique class of cell membrane-associated proteins contains a C-terminal glycosylphosphatidylinositol (GPI) moiety, and these lipoproteins associate with the outer leaflet of the membrane bilayer via the lipid chains of the GPI molecule. GPI-anchored proteins are functionally diverse including enzymes, cell adhesion molecules, receptors, complement regulatory proteins, immunoproteins and further proteins (e.g. prion protein) with unknown functions. ${ }^{1}$ It was shown that their biological functions were retained after exogenous addition to membranes ${ }^{2-5}$; therefore, semisynthetic lipoproteins containing diverse reporters in the headgroup of the lipid moiety can be valuable probes for investigating GPI-anchored proteins. $^{6-13}$ The GPI moiety directs the attached protein towards the extracellular space and locates the attached protein in special membrane microdomains, that is, lipid rafts. Because cholesterol maintains the dynamic lipid-lipoprotein associates of GPI-anchored proteins, sphingolipids and cholesterol, ${ }^{14,15}$ it is hypothesized that cholesteryl lipoproteins may also be accumulated in lipid rafts. ${ }^{16}$ Based on the finding that cholesteryl poly(ethylene glycol) ethers accumulate in cholesterol-rich membranes and their cytotoxicity is low, ${ }^{17}$ we have recently prepared a cholesterol derivative containing both a maleimide and a fluorescent reporter in the headgroup for protein conjugation and imaging, respectively. It was shown that after protein conjugation, the semisynthetic cholesteryl lipoprotein could be purified via a $\beta$-cyclodextrin inclusion complex that made the cholesterol moiety soluble in polar solvents. Then, the lipid excess- and detergent-free lipoprotein could be introduced into the cell membrane of live cells with minimal disruption of the membrane, and the fluorescence of the headgroup could be unambiguously assigned to the attached protein. ${ }^{18}$ In order to further study the membraneassociated cholesteryl lipoproteins, it is important to quantitate the lipoprotein incorporated into the cell membrane. It can be achieved by determining the radioactivity of cell membrane preparations or intact cells treated with a tritium labelled analogue of the anchor molecule. Herein, we report our results on the preparation and biological application of a tritium labelled cholesterol derivative designed for that purpose.

\footnotetext{
a Laboratory of Chemical Biology, Institute of Biochemistry, Biological Research Centre of the Hungarian Academy of Sciences, Temesvári krt. 62., 6726 Szeged, Hungary
}

${ }^{b}$ Research Group of Peptide Chemistry, Hungarian Academy of Sciences, Pázmány Péter sétány 1/a, 1117 Budapest, Hungary

${ }^{c}$ Department of Medicinal Chemistry, Szeged University, Dóm tér 8., 6720 Szeged, Hungary

*Correspondence to: Csaba Tömböly, Laboratory of Chemical Biology, Institute of Biochemistry, Biological Research Centre of the Hungarian Academy of Sciences, Temesvári krt. 62., 6726 Szeged, Hungary.

E-mail: tomboly@brc.hu

${ }^{+}$Current address: Institute of Biophysical Chemistry, Goethe University Frankfurt, Max-von-Laue str. 960438 Frankfurt, Germany 


\section{Experimental}

The purity of all reagents and solvents was analytical or the highest commercially available grade. Protected propargylglycine (Pra) and 5 -amiofluorescein were purchased from Bachem Feinchemicalen AG (Bubendorf, Switzerland), and coupling reagents and substituted di (ethylene glycols) were obtained from Calbiochem-Novabiochem AG (Läufelfingen, Switzerland). Other chemicals were from Sigma Ltd. (St. Louis, MO, USA). Tritium labelling was carried out in a self-designed vacuum manifold, ${ }^{19,20}$ and radioactivity was measured with a Packard Tri-Carb 2100 TR liquid scintillation analyser using Hionic-Fluor scintillation cocktail of PerkinElmer (Waltham, MA, USA). Analytical thin layer chromatography (TLC) was performed on $5 \times 10 \mathrm{~cm}$ glass plates precoated with silica gel $60 \mathrm{~F}_{254}$ (Merck, Darmstadt, Germany), spots were visualized with UV light, ninhydrin or phosphomolybdic acid, and radioactivity was detected with a Berthold LB511 linear TLC analyser. Flash chromatography was carried out on silica gel 60 (Sigma Ltd., St. Louis, MO, USA) using the indicated solvents. Analytical and semipreparative HPLC separations were performed with a Merck-Hitachi LaChrom system under the indicated conditions. Radio-HPLC was performed on a Jasco HPLC system equipped with a Packard Radiomatic 505 TR Flow Scintillation Analyser. NMR spectra were recorded on a Bruker Spectra DRX $500 \mathrm{MHz}$ spectrometer in the indicated solvent, and chemical shifts $(\delta)$ are reported in ppm after calibration to the solvent signals. Molecular weights of the compounds were determined by MS analysis on a Finnigan TSQ 7000 spectrometer equipped with an ESI source or on a Bruker reflex III MALDI-TOF spectrometer. In the case of MALDI-MS, 2,5-dihydroxybenzoic acid matrix was applied. Mass spectrometric measurements of $\left.{ }^{2} \mathrm{H}\right]$ piperidine were performed on a Micromass Q-TOF Premier mass spectrometer (Waters MSTechnologies, Manchester, UK) equipped with a nanoelectrospray ion source. The instrument was scanned in the mass range of $85-90$. Peak intensities were calculated for $[\mathrm{M}+\mathrm{H}]^{+}, \quad\left[\mathrm{M}\left({ }^{2} \mathrm{H}_{1}\right)+\mathrm{H}\right]^{+},\left[\mathrm{M}\left({ }^{2} \mathrm{H}_{2}\right)+\mathrm{H}\right]^{+}$and $\left[\mathrm{M}\left({ }^{2} \mathrm{H}_{3}\right)+\mathrm{H}\right]^{+}$ molecule ions by the Masslynx software. The measured peak intensities of mono-, di- and tri-deuterated ions were corrected by the calculated intensities of overlapping $\left[\mathrm{M}\left({ }^{13} \mathrm{C}\right)+\mathrm{H}\right]^{+},\left[\mathrm{M}\left({ }^{2} \mathrm{H}_{1},{ }^{13} \mathrm{C}\right)+\mathrm{H}\right]^{+}$and $[\mathrm{M}$ $\left.\left({ }^{2} \mathrm{H}_{2},{ }^{13} \mathrm{C}\right)+\mathrm{H}\right]^{+}$peaks, respectively. The effects of $\left[\mathrm{M}\left({ }^{13} \mathrm{C}_{2}\right)+\mathrm{H}\right]^{+}$and $[\mathrm{M}$ $\left.\left({ }^{2} \mathrm{H}_{1},{ }^{13} \mathrm{C}_{2}\right)+\mathrm{H}\right]^{+}$and ${ }^{15} \mathrm{~N}$ containing ions were neglected. Live cells treated with the fluorescent anchor were visualized with an Olympus IX81 confocal laser scanning microscope, and images were prepared by the FluoView 500 software.

\section{$\left[{ }^{2} \mathrm{H}\right]$ piperidine (1)}

An amount of $5 \mathrm{mg}$ of 4-bromopiperidine hydrobromide $(20 \mu \mathrm{mol})$ was dissolved in $400 \mu \mathrm{L}$ of $10 \mathrm{M} \mathrm{NaOH}$, and it was extracted with $1 \mathrm{~mL}$ of EtOAc. The organic phase was washed with brine, dried over $\mathrm{Na}_{2} \mathrm{SO}_{4}$ and evaporated at atmospheric pressure. The resulting amine was dissolved in $500 \mu \mathrm{L}$ of EtOAc, and it was reduced under 0.4 bar deuterium gas in the presence of $11 \mathrm{mg}$ of $\mathrm{Pd} / \mathrm{C}(10 \% \mathrm{Pd})$ catalyst. After $3 \mathrm{~h}$, the catalyst was filtered off, and the EtOAc solution was extracted with $1 \mathrm{M}$ $\left.\mathrm{HCl} .{ }^{2} \mathrm{H}\right]$ piperidine hydrochloride (1) was obtained after repeated evaporations from the acidic aqueous solution $(2 \mathrm{mg}, 82 \%) . R_{\mathrm{f}} 0.38(n-$ $\mathrm{BuOH}-\mathrm{AcOH}-\mathrm{H}_{2} \mathrm{O}$ 2:1:1); ESI-MS calcd for $\mathrm{C}_{5} \mathrm{H}_{10} \mathrm{DN} 86.10$, found 87.09 $[\mathrm{M}+\mathrm{H}]^{+}$.

\section{[ ${ }^{3}$ H]piperidine}

Tritium labelling was performed with $2.1 \mathrm{mg}$ of 4-bromopiperidine base $(12.9 \mu \mathrm{mol})$ dissolved in $1 \mathrm{~mL}$ of EtOAc in the presence of $5 \mathrm{mg}$ of $\mathrm{Pd} / \mathrm{C}$ $(10 \% \mathrm{Pd})$ catalyst. The reaction mixture was degassed prior to tritium reduction by a freeze-thaw cycle, and then it was stirred under 0.4 bar of tritium gas for $3 \mathrm{~h}$, followed by the filtration of the catalyst. The EtOAc solution was extracted with $1 \mathrm{M} \mathrm{HCl}$, and labile tritium was removed by repeated evaporations from $1 \mathrm{M} \mathrm{HCl}$ solution. Finally, $20.5 \mathrm{GBq}$ of $\left[{ }^{3} \mathrm{H}\right]$ piperidine hydrochloride was isolated as a white solid (s.a. $1.58 \mathrm{TBq} /$ $\mathrm{mmol}$ ), which was immediately used for the next step.

\section{$\left[{ }^{3} \mathrm{H}\right] \mathrm{N}$-nitrosopiperidine}

The solid $\left[{ }^{3} \mathrm{H}\right]$ piperidine hydrochloride $(20.5 \mathrm{GBq})$ was dissolved in $100 \mu \mathrm{L}$ of $1 \mathrm{M} \mathrm{HCl}$, and the solution was cooled into ice. Then, $250 \mathrm{mg}$ of $\mathrm{KNO}_{2}$ was added, and the reaction mixture was stirred at $0^{\circ} \mathrm{C}$ for $2 \mathrm{~h}$ followed by extraction with $\mathrm{Et}_{2} \mathrm{O}$. The ethereal solution was evaporated at atmospheric pressure yielding $17.8 \mathrm{GBq}(87 \%)$ of crude $\left[{ }^{3} \mathrm{H}\right] \mathrm{N}$ nitrosopiperidine. It was dissolved in $100 \mu \mathrm{L}$ THF and immediately used in the next step without further purification. $R_{f} 0.28$ (petroleum ether-acetone 9:1), 93\% radiochemical purity by radio-TLC.

\section{$\left[{ }^{3} \mathrm{H}\right] \mathrm{N}$-aminopiperidine (2)}

The solution of $17.8 \mathrm{GBq}$ of $\left[^{3} \mathrm{H}\right] \mathrm{N}$-nitrosopiperidine in $100 \mu \mathrm{L}$ of THF was transferred to a reaction vessel equipped with a condenser. A suspension of $4.5 \mathrm{mg}$ of $\mathrm{LiAlH}_{4}$ in $600 \mu \mathrm{L}$ of THF was added, and the reaction mixture was stirred under Ar at $60^{\circ} \mathrm{C}$ for $3.5 \mathrm{~h}$. After cooling, $100 \mu \mathrm{L}$ of $10 \mathrm{M} \mathrm{NaOH}$ was added, and it was stirred for further $20 \mathrm{~min}$. The resulting slurry was extracted with $\mathrm{Et}_{2} \mathrm{O}$, and the ethereal solution was extracted with $3 \mathrm{M}$ $\mathrm{HCl}$. The $\mathrm{HCl}$ solution was evaporated, and labile tritium was removed by repeated evaporations from water: ethanol (1:1), resulting in $8.4 \mathrm{GBq}$ $(47 \%)$ of solid $\left[{ }^{3} \mathrm{H}\right] \mathrm{N}$-aminopiperidine hydrochloride $(0.8 \mathrm{mg})$, which was used for the preparation of $\mathbf{8}$ without further purification. $R_{\mathrm{f}} 0.38$ ( $n$ $\mathrm{BuOH}-\mathrm{AcOH}-\mathrm{H}_{2} \mathrm{O}$ 2:1:1), 73\% radiochemical purity by radio-TLC, s.a. $1.33 \mathrm{TBq} / \mathrm{mmol}$.

\section{Cholesteryl (S)-1-(4-(2-(tert-butoxycarbonylamino)-2- carboxyethyl)-1H-1,2,3-triazol-1-yl)-13-oxo-3,6,9-trioxa-12- azahexadecan-16-oate (3)}

Boc-Pra-OH (60 mg, $281.4 \mu \mathrm{mol}$ ) was dissolved in $1 \mathrm{~mL}$ of dimethylformamide (DMF), and it was added to the solution of cholesteryl 1-azido-13-oxo-3,6,9trioxa-12-azahexadecan-16-oate ${ }^{18}(230 \mathrm{mg}, 335.0 \mu \mathrm{mol})$ in $\mathrm{CH}_{2} \mathrm{Cl}_{2}(4 \mathrm{~mL})$. Then, a solution of $\mathrm{L}(+)$-ascorbic acid sodium salt $(33.4 \mathrm{mg}, 168.8 \mu \mathrm{mol})$ and $\mathrm{CuSO}_{4} .5 \mathrm{H}_{2} \mathrm{O}(21.1 \mathrm{mg}, 84.4 \mu \mathrm{mol})$ in water $(4 \mathrm{~mL})$ was added. The reaction mixture was stirred for $8 \mathrm{~h}$ at room temperature, and then it was evaporated in vacuo. The crude product was purified by column chromatography $\left(\mathrm{CHCl}_{3}-\mathrm{MeOH}-\mathrm{AcOH}\right.$ 90:9:1) to give $230.4 \mathrm{mg}(91 \%)$ of pure 3 as a white solid. $R_{\mathrm{f}} 0.30\left(\mathrm{CHCl}_{3}-\mathrm{MeOH}-\mathrm{AcOH} 90: 9.5: 0.5\right) ; k^{\prime}=8.61\left(t_{\mathrm{R}}=12.5 \mathrm{~min} ; \mathrm{Vydac}\right.$ 214TP5410 C 4 column $(100 \times 4.6 \mathrm{~mm}, 5 \mu \mathrm{m})$, gradient elution with $1.8 \% / \mathrm{min}$ acetonitrile (ACN) $\left(0.08 \%(\mathrm{v} / \mathrm{v})\right.$ trifluoroacetic acid (TFA) in $\mathrm{H}_{2} \mathrm{O}(0.1 \%(\mathrm{v} / \mathrm{v})$ TFA) starting from $50 \% \mathrm{ACN}$, flow rate: $1 \mathrm{~mL} / \mathrm{min}, \lambda=216 \mathrm{~nm}) ;{ }^{1} \mathrm{H}$ NMR $\left(500 \mathrm{MHz}, \mathrm{CDCl}_{3}\right) \delta 7.63(\mathrm{~s}, 1 \mathrm{H}), 6.63(\mathrm{~s}, 1 \mathrm{H}), 5.61(\mathrm{~s}, 1 \mathrm{H}), 5.34(\mathrm{~d}, 1 \mathrm{H}$, $J=3.7 \mathrm{~Hz}), 4.58(\mathrm{~s}, 2 \mathrm{H}), 4.53(\mathrm{~m}, 1 \mathrm{H}), 4.47(\mathrm{~s}, 1 \mathrm{H}), 3.86(\mathrm{~s}, 2 \mathrm{H}), 3.60(\mathrm{~m}, 10 \mathrm{H})$, $3.42(\mathrm{q}, 2 \mathrm{H}, J=4.6 \mathrm{~Hz}), 3.28(\mathrm{~m}, 2 \mathrm{H}), 2.62(\mathrm{t}, 2 \mathrm{H}, J=6.7 \mathrm{~Hz}), 2.47(\mathrm{t}, 2 \mathrm{H}$, $J=6.7 \mathrm{~Hz}), 2.29(\mathrm{~d}, 2 \mathrm{H}, J=7.6 \mathrm{~Hz}), 1.98(\mathrm{~m}, 1 \mathrm{H}), 1.93(\mathrm{~m}, 1 \mathrm{H}), 1.83(\mathrm{~m}, 3 \mathrm{H})$, [1.62-1.00 (m, 18H): $1.57(3 \mathrm{H}), 1.52(1 \mathrm{H}), 1.48(2 \mathrm{H}), 1.40(1 \mathrm{H}), 1.32(1 \mathrm{H}), 1.30$ $(2 \mathrm{H}), 1.20(1 \mathrm{H}), 1.14(1 \mathrm{H}), 1.11(4 \mathrm{H}), 1.09(2 \mathrm{H})], 1.42(\mathrm{~s}, 9 \mathrm{H}), 1.00(\mathrm{~s}, 3 \mathrm{H})$, [0.99-0.86 (m, 3H): $0.94(2 \mathrm{H}), 0.88(1 \mathrm{H})], 0.90(\mathrm{~d}, 3 \mathrm{H}, J=6.4 \mathrm{~Hz}),[0.86(\mathrm{~d}, 3 \mathrm{H}$, $J=1.8 \mathrm{~Hz}), 0.84(\mathrm{~d}, 3 \mathrm{H}, J=1.8 \mathrm{~Hz})], 0.66(\mathrm{~s}, 3 \mathrm{H}) ;{ }^{13} \mathrm{C} \mathrm{NMR}\left(125 \mathrm{MHz}, \mathrm{CDCl}_{3}\right) \delta$ $172.7,172.1,155.7,142.7,139.7,124.1,122.7,80.0,74.5,70.7,70.5,70.3$, $70.1,69.9,69.5,56.8,56.2,53.3,50.5,50.1,42.4,39.8,39.6,39.3,38.2,37.1$, $36.7,36.3,35.9,32.0$ (2C), 31.0, 30.0, 28.5, 28.3, 28.12, 28.07, 27.8, 24.4, 23.9, 22.9, 22.7, 21.1, 19.4, 18.8, 12.0; MS(MALDI) calcd for $\mathrm{C}_{49} \mathrm{H}_{81} \mathrm{~N}_{5} \mathrm{O}_{10} 899.60$ found $900.44[\mathrm{M}+\mathrm{H}]^{+}$.

\section{Cholesteryl (S)-1-(4-(2-amino-2-carboxyethyl)-1H-1,2,3- triazol-1-yl)-13-oxo-3,6,9-trioxa-12-azahexadecan-16-oate} (4)

Compound 3 ( $200 \mathrm{mg}, 222.3 \mu \mathrm{mol}$ ) was dissolved in $2 \mathrm{~mL}$ of TFA $-\mathrm{CH}_{2} \mathrm{Cl}_{2}$ $1: 1$, and it was stirred for $30 \mathrm{~min}$. The solution was evaporated in vacuo to give $197.0 \mathrm{mg}(97 \%)$ of pure 4 trifluoroacetate as a pale yellow solid. $R_{\mathrm{f}}$ $0.12\left(\mathrm{CHCl}_{3}-\mathrm{MeOH}-\mathrm{AcOH} \quad 90: 9.5: 0.5\right) ; \quad k^{\prime}=4.15 \quad\left(t_{\mathrm{R}}=6.7 \mathrm{~min} ; \quad \mathrm{Vydac}\right.$ 214TP5410 C4 column $(100 \times 4.6 \mathrm{~mm}, 5 \mu \mathrm{m})$, gradient elution with $1.8 \% / \mathrm{min}$ ACN $\left(0.08 \%(\mathrm{v} / \mathrm{v})\right.$ TFA) in $\mathrm{H}_{2} \mathrm{O}(0.1 \%(\mathrm{v} / \mathrm{v})$ TFA) starting from $50 \%$ ACN, flow rate: $1 \mathrm{~mL} / \mathrm{min}, \lambda=216 \mathrm{~nm}$ ); MS(MALDI) calcd for $\mathrm{C}_{44} \mathrm{H}_{73} \mathrm{~N}_{5} \mathrm{O}_{8} 799.55$, found $800.48[\mathrm{M}+\mathrm{H}]^{+}$. 


\section{Cholesteryl (S)-1-(4-(2-carboxy-2-(3-(maleimido) propanamido)ethyl)-1H-1,2,3-triazol-1-yl)-13-oxo-3,6,9- trioxa-12-azahexadecan-16-oate (5)}

The amino acid derivative $\mathbf{4}$ (140 mg, $153.2 \mu \mathrm{mol})$ and N-ethyldiisopropylamine (DIEA) $(61 \mu \mathrm{L}, 350.4 \mu \mathrm{mol})$ were dissolved in $\mathrm{MeOH}(4 \mathrm{~mL})$, and 3maleimidopropionic acid $\mathrm{N}$-hydroxysuccinimide ester $(56.0 \mathrm{mg}, 210.4 \mu \mathrm{mol})$ was added. The mixture was stirred for $3 \mathrm{~h}$ at room temperature, and then it was evaporated in vacuo. The crude product was purified by column chromatography $\left(\mathrm{CHCl}_{3}-\mathrm{MeOH}-\mathrm{AcOH} 85: 14: 1\right)$ to give $118 \mathrm{mg}$ (81\%) of pure 5 as a white solid. $R_{\mathrm{f}} 0.43\left(\mathrm{CHCl}_{3}-\mathrm{MeOH}-\mathrm{AcOH} 80: 19: 1\right) ; k^{\prime}=6.53$ $\left(t_{R}=9.8 \mathrm{~min} ; \mathrm{Vydac} 214 \mathrm{TP} 5410 \mathrm{C} 4\right.$ column $(100 \times 4.6 \mathrm{~mm}, 5 \mu \mathrm{m})$, gradient elution with $1.8 \% / \mathrm{min} \mathrm{ACN}\left(0.08 \%(\mathrm{v} / \mathrm{v})\right.$ TFA) in $\mathrm{H}_{2} \mathrm{O}(0.1 \%(\mathrm{v} / \mathrm{v})$ TFA) starting from $50 \%$ ACN, flow rate: $1 \mathrm{~mL} / \mathrm{min}, \lambda=216 \mathrm{~nm}) ;{ }^{1} \mathrm{H} \mathrm{NMR}\left(500 \mathrm{MHz}, \mathrm{CDCl}_{3}\right) \delta$ $7.70(\mathrm{~s}, 1 \mathrm{H}), 7.02(\mathrm{brs}, 1 \mathrm{H}), 6.68(\mathrm{~s}, 2 \mathrm{H}), 6.63(\mathrm{brs}, 1 \mathrm{H}), 5.34(\mathrm{~d}, 1 \mathrm{H}, J=3.7 \mathrm{~Hz})$, 4.77 (brs, 1H), $4.57(\mathrm{~m}, 2 \mathrm{H}), 4.49(\mathrm{~s}, 1 \mathrm{H}), 3.82(\mathrm{~m}, 4 \mathrm{H}), 3.61(\mathrm{~m}, 10 \mathrm{H}), 3.42(\mathrm{q}$, $2 \mathrm{H}, J=4.6 \mathrm{~Hz}), 3.24(\mathrm{~m}, 2 \mathrm{H}), 2.62(\mathrm{t}, 2 \mathrm{H}, J=6.7 \mathrm{~Hz}), 2.58(\mathrm{t}, 2 \mathrm{H}, J=6.3 \mathrm{~Hz})$, $2.47(\mathrm{t}, 2 \mathrm{H}, J=6.7 \mathrm{~Hz}), 2.30(\mathrm{~d}, 2 \mathrm{H}, J=7.6 \mathrm{~Hz}), 2.01(\mathrm{~m}, 1 \mathrm{H}), 1.94(\mathrm{~m}, 1 \mathrm{H})$, $1.83(\mathrm{~m}, 3 \mathrm{H}),[1.62-1.03(\mathrm{~m}, 18 \mathrm{H}): 1.57(3 \mathrm{H}), 1.52(1 \mathrm{H}), 1.48(2 \mathrm{H}), 1.40(1 \mathrm{H})$, $1.32(1 \mathrm{H}), 1.30(2 \mathrm{H}), 1.20(1 \mathrm{H}), 1.14(1 \mathrm{H}), 1.11(4 \mathrm{H}), 1.09(2 \mathrm{H})], 1.00(\mathrm{~s}, 3 \mathrm{H})$, [0.99-0.86 (m, 3H): $0.94(2 \mathrm{H}), 0.88(1 \mathrm{H})], 0.90(\mathrm{~d}, 3 \mathrm{H}, J=6.4 \mathrm{~Hz}),[0.86(\mathrm{~d}, 3 \mathrm{H}$, $J=1.8 \mathrm{~Hz}), 0.84(\mathrm{~d}, 3 \mathrm{H}, J=1.8 \mathrm{~Hz})], 0.66(\mathrm{~s}, 3 \mathrm{H}) ;{ }^{13} \mathrm{C} \mathrm{NMR}\left(125 \mathrm{MHz}, \mathrm{CDCl}_{3}\right) \delta$ $172.80,172.7,172.2,170.7,169.9,142.7,139.8,134.3,124.3,122.8,74.5$, $70.6,70.5,70.3,70.0,69.9,69.4,56.8,56.3,53.5,50.5,50.1,42.4,39.8,39.6$, $39.4,38.2,37.1,36.7,36.3,35.9,34.6,34.3,32.0$ (2C), 31.0, 30.0, 28.4, 28.1 (2C), 27.9, 24.4, 24.0, 23.0, 22.7, 21.2, 19.4, 18.8, 12.0; MS(MALDI) calcd for $\mathrm{C}_{51} \mathrm{H}_{78} \mathrm{~N}_{6} \mathrm{O}_{11} 950.57$, found $951.45[\mathrm{M}+\mathrm{H}]^{+}$.

\section{Fluorescent cholesterol anchor 6}

The maleimide derivative $\mathbf{5}$ (50 mg, $52.6 \mu \mathrm{mol}$ ) and 1-[Bis(dimethylamino) methylene]-1 $\mathrm{H}$-1,2,3-triazolo[4,5-b]pyridinium 3-oxid hexafluorophosphate (HATU) $(20.0 \mathrm{mg}, 52.6 \mu \mathrm{mol})$ were dissolved in THF $(5 \mathrm{~mL})$, and DIEA $(9.1 \mu \mathrm{L}, 52.6 \mu \mathrm{mol})$ was added to the solution. It was stirred for $5 \mathrm{~min}$ followed by the addition of fluorescein amine $(54.8 \mathrm{mg}, 157.8 \mu \mathrm{mol})$ in THF $(2 \mathrm{~mL})$. The reaction mixture was stirred for $16 \mathrm{~h}$ at room temperature, and then it was evaporated in vacuo; the crude product was purified by column chromatography $\left(\mathrm{CHCl}_{3}-\mathrm{MeOH}-\mathrm{AcOH}\right.$ 90:9:1) to give $37.0 \mathrm{mg}$ (55\%) of pure 6 as an orange solid. $R_{\mathrm{f}} 0.41\left(\mathrm{CHCl}_{3}-\mathrm{MeOH}-\mathrm{AcOH} 90: 9: 1\right) ; k^{\prime}$ $=8.53\left(t_{R}=12.4 \mathrm{~min}\right.$; Vydac $214 \mathrm{TP} 5410 \mathrm{C} 4$ column $(100 \times 4.6 \mathrm{~mm}, 5 \mu \mathrm{m})$, gradient elution with $1.8 \% / \mathrm{min} \mathrm{ACN}\left(0.08 \%(\mathrm{v} / \mathrm{v})\right.$ TFA) in $\mathrm{H}_{2} \mathrm{O}(0.1 \%(\mathrm{v} / \mathrm{v})$ TFA) starting from $50 \%$ ACN, flow rate: $1 \mathrm{~mL} / \mathrm{min}, \lambda=216 \mathrm{~nm}) ;{ }^{1} \mathrm{H}$ NMR $\left(500 \mathrm{MHz},\left(\mathrm{CD}_{3}\right)_{2} \mathrm{SO}\right) \delta 10.30(\mathrm{~s}, 1 \mathrm{H}), 10.21$ (brs, 2H), $8.43(\mathrm{~s}, 1 \mathrm{H}), 8.32(\mathrm{~d}$, $1 \mathrm{H}, J=5.7 \mathrm{~Hz}), 7.89(\mathrm{t}, 1 \mathrm{H}, J=5.4 \mathrm{~Hz}), 7.79(\mathrm{~s}, 1 \mathrm{H}), 7.74(\mathrm{~d}, 1 \mathrm{H}, J=8.3 \mathrm{~Hz})$, $7.15(\mathrm{~d}, 1 \mathrm{H}, J=8.3 \mathrm{~Hz}), 6.97(\mathrm{~s}, 2 \mathrm{H}), 6.90(\mathrm{~s}, 2 \mathrm{H}), 6.87(\mathrm{dd}, 2 \mathrm{H}, J=8.6,2.6 \mathrm{~Hz})$, $6.73(\mathrm{dd}, 2 \mathrm{H}, J=8.6,1.6 \mathrm{~Hz}), 5.31(\mathrm{~d}, 1 \mathrm{H}, J=3.2 \mathrm{~Hz}), 5.09(\mathrm{q}, 1 \mathrm{H}, J=5.8 \mathrm{~Hz})$, $4.47(\mathrm{t}, 2 \mathrm{H}, J=5.1 \mathrm{~Hz}), 4.42(\mathrm{~m}, 1 \mathrm{H}), 3.92(\mathrm{~m}, 2 \mathrm{H}), 3.77(\mathrm{t}, 2 \mathrm{H}, J=5.1 \mathrm{~Hz})$, 3.45 (brs, $10 \mathrm{H}), 3.38(\mathrm{q}, 2 \mathrm{H}, J=5.7 \mathrm{~Hz}), 3.12(\mathrm{~m}, 1 \mathrm{H}), 3.05(\mathrm{dd}, 1 \mathrm{H}, J=14.9$, $7.1 \mathrm{~Hz}), 2.71(\mathrm{~m}, 2 \mathrm{H}), 2.43(\mathrm{t}, 2 \mathrm{H}, J=6.7 \mathrm{~Hz}), 2.34(\mathrm{t}, 2 \mathrm{H}, J=6.7 \mathrm{~Hz}), 2.22(\mathrm{~d}$, $2 \mathrm{H}, J=7.6 \mathrm{~Hz}), 1.92(\mathrm{~m}, 1 \mathrm{H}), 1.85(\mathrm{~m}, 1 \mathrm{H}), 1.76(\mathrm{~m}, 3 \mathrm{H}),[1.60-0.92$ $(\mathrm{m}, 21 \mathrm{H}): 1.48(2 \mathrm{H}), 1.52(1 \mathrm{H}), 1.47(1 \mathrm{H}), 1.44(2 \mathrm{H}), 1.39(1 \mathrm{H}), 1.28$ $(3 \mathrm{H}), 1.17(1 \mathrm{H}), 1.13(1 \mathrm{H}), 1.11(4 \mathrm{H}), 1.02(1 \mathrm{H}), 1.00(1 \mathrm{H}), 0.97(1 \mathrm{H})$, $0.96(1 \mathrm{H}), 0.91(1 \mathrm{H})], 0.95(\mathrm{~s}, 3 \mathrm{H}), 0.87(\mathrm{~d}, 3 \mathrm{H}, J=6.4 \mathrm{~Hz}), 0.84(\mathrm{~d}, 3 \mathrm{H}$, $J=2.0 \mathrm{~Hz}), 0.81(\mathrm{~d}, 3 \mathrm{H}, J=2.0 \mathrm{~Hz}), 0.65(\mathrm{~s}, 3 \mathrm{H}, 18-\mathrm{H}) ;{ }^{13} \mathrm{C} \mathrm{NMR}$ $\left(125 \mathrm{MHz},\left(\mathrm{CD}_{3}\right)_{2} \mathrm{SO}\right) \delta 171.5,171.2,171.0,170.6,169.3,168.5,159.2$, $152.7,146.5,142.8,142.1,139.2,134.1,129.6,125.9,125.5,125.3$, $123.1,121.9,116.9,114.0,109.1,101.3,83.8,72.8,69.8,69.7$ (2C), $69.6,69.3,69.2,56.1,55.9,55.4,49.5,49.4,42.1,39.1,39.0,38.8$, $37.7,36.6,36.1,35.7,35.0,34.9,34.5,31.8,31.1,29.7,29.2,27.9$, 27.7, 27.4, 27.2, 23.9, 23.3, 22.6, 22.4, 20.9, 19.0, 18.6, 11.7; MS(MALDI) calcd for $\mathrm{C}_{71} \mathrm{H}_{89} \mathrm{~N}_{7} \mathrm{O}_{15} 1279.64$, found $1280.56[\mathrm{M}+\mathrm{H}]^{+}$.

\section{Cholesterol anchor 7}

The maleimide derivative 5 ( $25 \mathrm{mg}, 26.3 \mu \mathrm{mol}), \mathrm{N}$-hydroxybenzotriazole (HOBt) $(3.6 \mathrm{mg}, 26.3 \mu \mathrm{mol})$ and $\mathrm{N}^{\mathrm{N}} \mathrm{N}^{\prime}$-diisopropylcarbodiimide (DIC) $(4 \mu \mathrm{L}$, $26.3 \mu \mathrm{mol})$ were dissolved in DMF $(2 \mathrm{~mL})$. Then, $\mathrm{N}$-aminopiperidine (3.1 $\mu \mathrm{L}, 29 \mu \mathrm{mol}$ ) was added, and the solution was stirred for $16 \mathrm{~h}$ at room temperature. It was evaporated in vacuo, and the crude product was purified by HPLC on a Discovery Bio Wide Pore $C_{5}$ column to give $16 \mathrm{mg}(59 \%)$ of pure 7 as a white solid. $R_{\mathrm{f}} 0.45\left(\mathrm{CHCl}_{3}-\mathrm{MeOH}-\mathrm{TEA}\right.$ 90:9.9:0.1); $k^{\prime}=4.31 \quad\left(t_{R}=6.9 \mathrm{~min} ; \quad V y d a c \quad 214 \mathrm{TP} 5410 \quad\right.$ C4 column $(100 \times 4.6 \mathrm{~mm}, 5 \mu \mathrm{m})$, gradient elution with $1.5 \% / \mathrm{min}$ ACN $(0.08 \%(\mathrm{v} / \mathrm{v})$ TFA) in $\mathrm{H}_{2} \mathrm{O}(0.1 \%(\mathrm{v} / \mathrm{v})$ TFA) starting from $60 \% \mathrm{ACN}$, flow rate: $1 \mathrm{~mL} / \mathrm{min}, \lambda=216 \mathrm{~nm}) ;{ }^{1} \mathrm{H}$ NMR $\left(500 \mathrm{MHz}, \mathrm{CDCl}_{3}\right) \delta 7.63(\mathrm{~s}, 1 \mathrm{H}), 7.08(\mathrm{~s}$, $1 \mathrm{H}), 6.68(\mathrm{~s}, 2 \mathrm{H}), 6.60(\mathrm{~s}, 1 \mathrm{H}), 6.48(\mathrm{~s}, 1 \mathrm{H}), 5.34(\mathrm{~d}, 1 \mathrm{H}, J=3.7 \mathrm{~Hz}), 4.87$ (brs, 1H), $4.55(\mathrm{~m}, 2 \mathrm{H}), 4.46(\mathrm{~s}, 1 \mathrm{H}), 3.83(\mathrm{~s}, 4 \mathrm{H}), 3.61$ (brs, 10H), 3.42 (q, $2 \mathrm{H}, J=4.6 \mathrm{~Hz}), 3.20(\mathrm{~m}, 2 \mathrm{H}), 2.73(\mathrm{~m}, 4 \mathrm{H}), 2.62(\mathrm{t}, 2 \mathrm{H}, J=6.7 \mathrm{~Hz}), 2.58(\mathrm{t}$, $2 \mathrm{H}, J=6.7 \mathrm{~Hz}), 2.47(\mathrm{t}, 2 \mathrm{H}, J=7.6 \mathrm{~Hz}), 2.29(\mathrm{~d}, 2 \mathrm{H}), 1.99(\mathrm{~m}, 1 \mathrm{H}), 1.96(\mathrm{~m}$, $1 \mathrm{H}), 1.83(\mathrm{~m}, 3 \mathrm{H}), 1.71(\mathrm{~m}, 4 \mathrm{H}),[1.58-1.01(\mathrm{~m}, 20 \mathrm{H}): 1.55(3 \mathrm{H}), 1.50(1 \mathrm{H})$, $1.47(2 \mathrm{H}), 1.43(\mathrm{~m}, 3 \mathrm{H}), 1.32(1 \mathrm{H}), 1.30(2 \mathrm{H}), 1.23(1 \mathrm{H}), 1.14(1 \mathrm{H}), 1.11$ (4H), $1.09(1 \mathrm{H}), 1.05(1 \mathrm{H})], 1.00(\mathrm{~s}, 3 \mathrm{H}),[0.96-0.83(\mathrm{~m}, 3 \mathrm{H}): 0.96(1 \mathrm{H}), 0.92$ $(1 \mathrm{H}), 0.90(1 \mathrm{H})], 0.88(\mathrm{~d}, 3 \mathrm{H}, J=6.4 \mathrm{~Hz}),[0.86(\mathrm{~d}, 3 \mathrm{H}, J=1.8 \mathrm{~Hz}), 0.84(\mathrm{~d}$, $3 \mathrm{H}, J=1.8 \mathrm{~Hz})], 0.66(\mathrm{~s}, 3 \mathrm{H}) ;{ }^{13} \mathrm{C}$ NMR $\left(125 \mathrm{MHz}, \mathrm{CDCl}_{3}\right) \delta 172.7,172.2$, $170.7,169.9,156.8142 .7,139.8,134.3,124.3,122.8,74.5,70.6,70.5,70.3$, $70.0,69.9,69.4,63.4,56.8,56.3,53.6,50.5,50.1,42.4,39.8,39.6,39.4$, $38.2,37.1,36.7,36.3,35.9,34.6,34.3,32.0$ (2C), 31.0, 30.0, 28.4, 28.14, $28.12,27.9,27.2,24.4,24.0,23.5,23.0,22.7,21.2,19.4,18.8,12.0 ; \mathrm{MS}$ (MALDI) calcd for $\mathrm{C}_{56} \mathrm{H}_{88} \mathrm{~N}_{8} \mathrm{O}_{10} 1032.66$, found $1033.58[\mathrm{M}+\mathrm{H}]^{+}$.

\section{$\left[{ }^{3} \mathrm{H}\right]$ cholesterol anchor 8}

The maleimide derivative 5 ( 1 mg, $1 \mu \mathrm{mol})$ was dissolved in DMF (300 $\mu \mathrm{L}$ ) containing HOBt $(0.13 \mathrm{mg}, 1 \mu \mathrm{mol})$, DIC $(0.14 \mu \mathrm{L}, 1 \mu \mathrm{mol})$ and DIEA $(0.09 \mu \mathrm{L}, 0.5 \mu \mathrm{mol})$. Then, $\left[{ }^{3} \mathrm{H}\right] \mathrm{N}$-aminopiperidine $(100 \mathrm{MBq})$ dissolved in $24 \mu \mathrm{L}$ of DMF was added, and the solution was stirred for $16 \mathrm{~h}$ at room temperature. It was evaporated in vacuo; the crude product was dissolved in HFIP, and it was purified by HPLC on a Vydac 214TP5410 column to give $17.4 \mathrm{MBq}$ of pure 8. $k^{\prime}=4.31 \quad\left(t_{\mathrm{R}}=6.9 \mathrm{~min}\right.$; Vydac 214TP5410 C4 column $(100 \times 4.6 \mathrm{~mm}, 5 \mu \mathrm{m})$, gradient elution with $1.5 \% / \mathrm{min}$ ACN $\left(0.08 \%(\mathrm{v} / \mathrm{v})\right.$ TFA) in $\mathrm{H}_{2} \mathrm{O}(0.1 \%(\mathrm{v} / \mathrm{v})$ TFA) starting from $60 \%$ ACN, flow rate: $1 \mathrm{~mL} / \mathrm{min}, \lambda=216 \mathrm{~nm}$ ), s.a. $1.37 \mathrm{TBq} / \mathrm{mmol}$.

\section{Cell culture}

SH-SY5Y (ATCC: CRL-2266) human neuroblastoma cells were used to test the membrane association potency of $\mathbf{6}$ and $\mathbf{8}$. Cells were cultured in DMEM (Sigma Ltd., St. Louis, MO, USA) medium containing 10\% FCS, L-glutamine $(2 \mathrm{mM})$, gentamycin $(160 \mu \mathrm{g} / \mathrm{mL}), 1 \mathrm{mM}$ pyruvate and nonessential amino acids (Sigma Ltd., St. Louis, MO, USA). The cell culture was maintained at $37^{\circ} \mathrm{C}$ in a humidified atmosphere with $5 \% \mathrm{CO}_{2}$.

\section{Live cell imaging with confocal laser scanning microscopy}

Twenty thousand cells per well were plated on an 8-well Lab-Tek II Chambered cover glass. After $48 \mathrm{~h}$ incubation at $37^{\circ} \mathrm{C}$, cells were treated with $1 \mu \mathrm{M}$ of 6 dissolved in serum-free medium containing $10 \mu \mathrm{M} \beta$ cyclodextrin for $30 \mathrm{~min}$. Cells treated with serum-free medium for $30 \mathrm{~min}$ were used as a negative control. After treatment and incubation, cells were washed with serum-free medium, and the nuclei of the cells were stained for $5 \mathrm{~min}$ with DRAQ5. Sequential excitation was applied for fluorescein and DRAQ5. Fluorescein was excited with an Ar ion laser $(488 \mathrm{~nm})$, and emitted photons were collected through a BA 505-525 nm filter; DRAQ5 was excited with a He-Ne laser at $633 \mathrm{~nm}$, and emitted photons were collected through a BA $660 \mathrm{~nm}$ filter.

\section{Membrane incorporation of $\left[{ }^{3} \mathrm{H}\right]$ cholesterol anchor 8}

$10^{6}$ cells per well were plated on a 24-well plate, and after $12 \mathrm{~h}$ incubation at $37^{\circ} \mathrm{C}$, cells were treated with $1-100 \mathrm{nM}$ of $\mathbf{2}$ or $\mathbf{8}$ dissolved in serum-free medium containing $10 \mu \mathrm{M} \beta$-cyclodextrin for 10 and $30 \mathrm{~min}$. Then, cells were washed with serum-free medium and trypsinized. The resulting cell suspensions were filtered on a GF/B filter (Whatman) using a 24-well cell harvester (Brandel) and washed with phosphate-buffered saline (PBS) pH7.4. Filter discs containing the cells were immersed into Ultima Gold XR scintillation cocktail, and they were counted in glass vials with a 
Packard Tri-Carb 2100 TR LSA. Cells treated with serum-free medium for 30 min were used for background counting.

\section{Results and discussion}

Our earlier studies revealed that a maleimide functionalized fluorescent cholesterol derivative is capable of anchoring proteins to the plasma membrane of live cells. ${ }^{18}$ The headgroup of the anchor molecule contains a diethylene glycol residue, which increases the polarity of the molecule and exposes the attached protein towards the extracellular space. When applied in a form of $\beta$-cyclodextrin inclusion complex, the headgroup fluorescence of the cholesterol anchor can be used for the unambiguous visualization of the attached protein after cell membrane delivery. In order to determine the membrane concentration of the cholesterol anchor, a tritium labelled analogue (8) was designed. By using this compound, the quantitation of the membrane incorporated protein anchor can be achieved by measuring the radioactivity of cell membrane preparations or intact cells. For this purpose, a defined position of the radioactive label is crucial. Although $\left[1,2-{ }^{3} \mathrm{H}\right]$ cholesterol complexed with $\beta$-cyclodextrin was reported to use for the fast enrichment of cell membranes with labelled cholesterol, ${ }^{21}$ and it was also used in studying the cyclodextrin-mediated cholesterol transfer between different lipid layers ${ }^{22}$; cholesterol labelling is not appropriate for our purposes. The headgroup of the cholesterol anchor can be enzymatically removed via the hydrolysis of the cholesteryl ester, and as a result the labelled cholesterol, moiety remains in the cell membrane and the unlabelled headgroup is released to the cell culture medium. In this case, the membrane radioactivity is not affected by the presence or lack of the headgroup, and the process cannot be detected, resulting in overestimation of the amount of the membrane-associated cholesterol anchor. In contrast, if the tritium label is incorporated into the headgroup of the anchor molecule, enzymatic removal of the headgroup will result in decreasing radioactivity of the cell membrane preparation and also emerging the labelled headgroup in the cell culture medium.

In order to introduce the radioactive label into the cholesterol anchor headgroup, a convergent synthetic strategy was applied. The tritium label was incorporated into a small molecule that was coupled to an anchor fragment bearing both the cholesterol residue and the bulk of the headgroup. The advantage of this approach is that tritium incorporation either into the cholesterol moiety or into other parts of the headgroup is excluded. The cholesterol amphiphile $\mathbf{4}$ is an appropriate intermediate, because primary amine and carboxylic acid functions in the headgroup are available for the introduction of a radiolabelled or a fluorescent tag, and a protein conjugation function as well. It was prepared from $N^{\alpha}$-tBoc-protected propargylglycine and cholesteryl 1-azido-13-oxo-3,6,9-trioxa-12-azahexadecan-16-oate via a dipolar cycloaddition in the presence of $\mathrm{CuSO}_{4}$ and sodium ascorbate (Scheme 1). The $N^{\alpha}$-tBoc-protecting group was removed by TFA in DCM, and the amine $\mathbf{4}$ was liberated in situ with DIEA during the next step. Then, $\mathbf{4}$ was used for the preparation of two constitutionally related tritium or fluorescein labelled anchors. First, the thiol reactive maleimide group was coupled to the amine $\mathbf{4}$, because this way, the co-existence of a Michael acceptor and a primary amine in an intermediate compound is excluded that otherwise could lead to self-addition under the basic conditions of the introduction of the labelling tag. It was found that the amino function of $\mathbf{4}$ reacts faster with an $\mathrm{N}$-hydroxysuccinimide ester than with a maleimide, and accordingly, the $\mathrm{N}$-acylation of $\mathbf{4}$ with 3-maleimidopropionic acid<smiles>CC(C)CCCC1CCC2C3CC=C4CC(OC(=O)CCC(=O)NC(C)(C)CCC(C)C)CCC4(C)C3CCC12C</smiles>
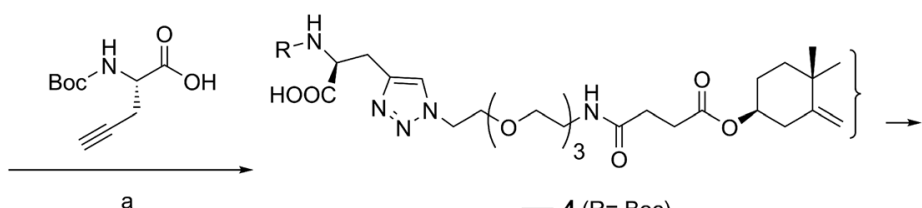<smiles>CCCCC(=O)OCCN1C(=O)C=CC1=O</smiles><smiles>CC(C)CCN1C(=O)C=CC1=O</smiles>

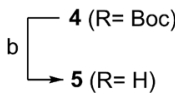

c<smiles>CN=CCC(N)O</smiles><smiles>CCCCCC(C)Cn1cc(C)nn1</smiles><smiles>CC(C)(C)NC(=O)CCC(=O)OC1CCC2(C)CC=C(C1)C2(C)C</smiles><smiles>CC(C)(Cn1cc(C[C@@H](NC(=O)CCN2C(=O)C=CC2=O)C(=O)Nc2ccc(-c3c4ccc(=O)cc-4oc4cc(O)ccc34)c(C(=O)O)c2)nn1)CC(C)(C)NC(=O)CCC(=O)OC1CCC2C=CC(C2)C1</smiles>

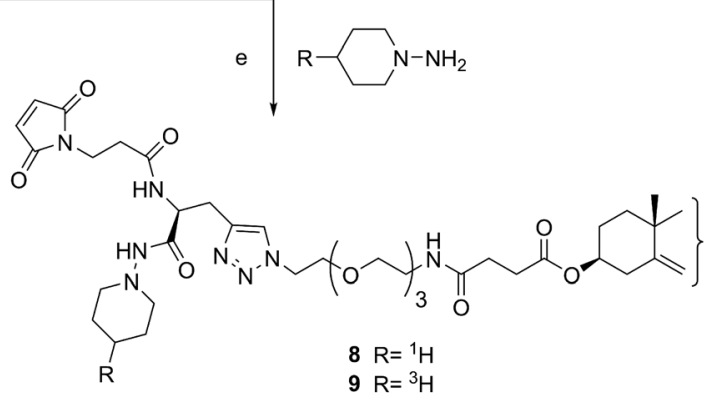

Scheme 1. Preparation of the fluorescein and tritium labelled cholesterol anchors: (a) $\mathrm{NaAsc}, \mathrm{CuSO}_{4} \cdot 5 \mathrm{H}_{2} \mathrm{O}, \mathrm{DMF}^{-} \mathrm{CH}_{2} \mathrm{Cl}{ }_{2}-\mathrm{H}_{2} \mathrm{O}(1: 4: 4), \mathrm{RT}, 8 \mathrm{~h}$; (b) TFA-CH $\mathrm{Cl}_{2}(1: 1), \mathrm{RT}$, 30 min; (c) 2 eq DIEA, MeOH, RT, 3 h; (d) HATU, DIEA, THF, RT, 16 h; (e) HOBt, DIC, DMF, RT, 16 h. 
$\mathrm{N}$-hydroxysuccinimide ester in DMF resulted in $\mathbf{5}$. This maleimide serves as a possible protein conjugation function in biological applications. In order to avoid the addition of nucleophiles to the maleimide of $\mathbf{5}$, an aniline and an alkyl-hydrazine-both are less basic than primary amines-were used to introduce the fluorescein and the tritium label, respectively. In a preliminary study, it turned out that the aniline moiety of 5-aminofluorescein did not react with the Michael acceptor of $\mathrm{N}$-(2-maleimidoethyl)2-aminopent-4-ynamide under the coupling condition applied for the preparation of $\mathbf{6}$. Then, the fluorescent anchor $\mathbf{6}$ was obtained by coupling of the carboxyl group of 5 with 5aminofluorescein in the presence of the coupling agent HATU. The NMR structure investigation revealed that 5aminofluorescein was conjugated to the carboxylic function of $\mathbf{5}$.

Based on earlier observations on isotopomer radioligands, ${ }^{21-23}$ the tritium label was introduced via an aliphatic compound instead of aniline to increase the radiolytic stability of the labelled anchor molecule. $\mathrm{N}$-Aminopiperidine was chosen for that purpose, because it is less basic than cyclohexylamine, ${ }^{24,25}$ and after $\mathrm{N}$-acylation, it results in stable hydrazides. Coupling of tritium labelled $\mathrm{N}$-aminopiperidine to the carboxylic acid function of $\mathbf{5}$ results in an anchor molecule possessing a headgroup homologous to that of $\mathbf{6}$, and this way, the

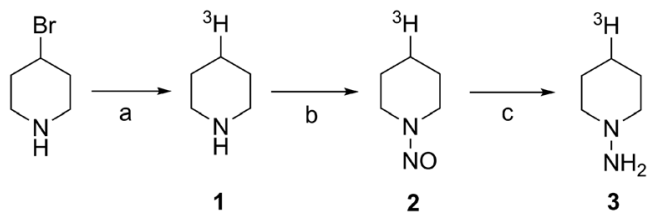

Scheme 2. Preparation of $\left[{ }^{3} \mathrm{H}\right] \mathrm{N}$-aminopiperidine: (a) $\mathrm{Pd} / \mathrm{C}(10 \% \mathrm{Pd}),{ }^{2} \mathrm{H}_{2}$ (1) or ${ }^{3} \mathrm{H}_{2}$ (2), EtOAc, RT, $3 \mathrm{~h}$; (b) $\mathrm{KNO}_{2}, 1 \mathrm{M} \mathrm{HCl}, 0{ }^{\circ} \mathrm{C}, 2 \mathrm{~h}$; (c) $\mathrm{LiAlH}_{4}, \mathrm{THF}, \mathrm{Ar}$ atm, $60{ }^{\circ} \mathrm{C}, 3.5 \mathrm{~h}$.

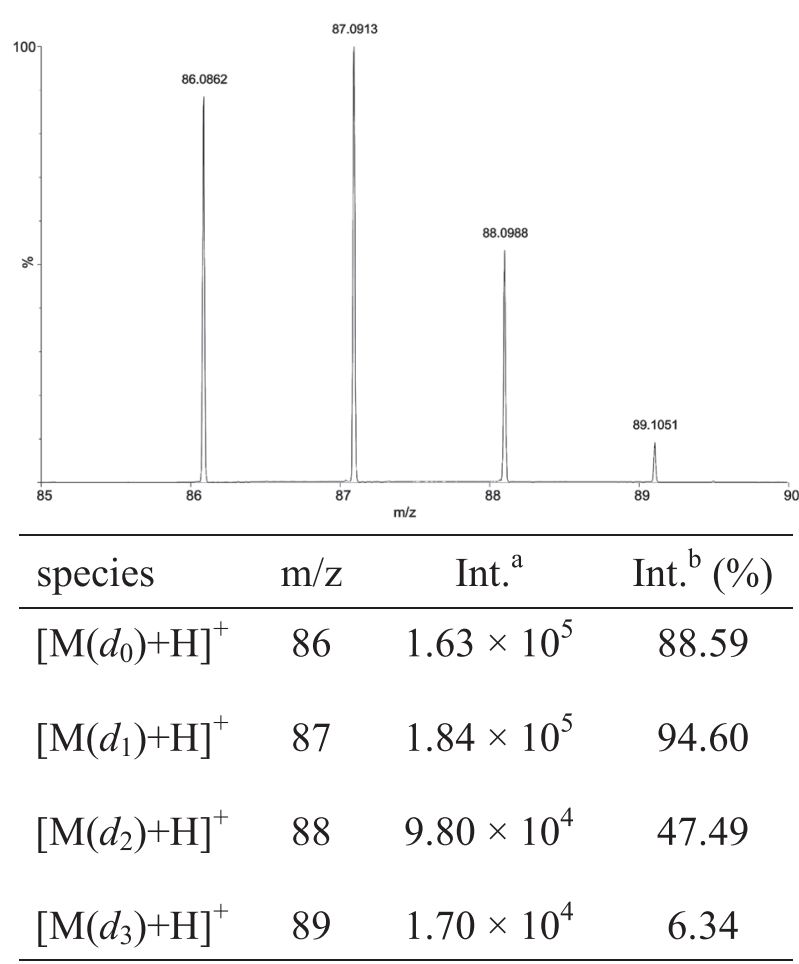

Figure 1. ESI mass spectrum of $\left[{ }^{2} \mathrm{H}\right]$ piperidine and the abundance of the isotopologues; ${ }^{a}$ measured intensity, ${ }^{b}$ relative intensity corrected with the natural abundance of ${ }^{2} \mathrm{H},{ }^{13} \mathrm{C}$. nonspecific tritium incorporation into the cholesterol moiety or into the headgroup is not feasible. Before preparing the tritium labelled anchor by $\mathrm{N}$-acylation of $\left[{ }^{3} \mathrm{H}\right] \mathrm{N}$-aminopiperidine with $\mathbf{5}$, the unlabelled isotopologue $\mathbf{7}$ was prepared in order to optimize the reaction conditions and carry out NMR measurements. For the preparation of the tritium labelled $\mathrm{N}$-aminopiperidine, 4-bromopiperidine was considered as a precursor in a deuterium labelling experiment (Scheme 2). The 4-bromopiperidine base was liberated from the hydrobromide salt, and it was reduced with deuterium gas in the presence of $\mathrm{Pd} / \mathrm{C}(10 \% \mathrm{Pd})$ catalyst. The resulting $\left[{ }^{2} \mathrm{H}\right]$ piperidine hydrochloride was analysed by TLC and ESI-MS. The separation of 4-bromopiperidine and piperidine was achieved on silica gel $60 \mathrm{~F}_{254}$ glass plates in $n-\mathrm{BuOH}-\mathrm{AcOH}-\mathrm{H}_{2} \mathrm{O}$ 2:1:1, as their $R_{\mathrm{f}}$ values were found to be 0.48 and 0.38 , respectively. The TLC analysis of the crude $\left[{ }^{2} \mathrm{H}\right]$ piperidine hydrochloride revealed that the conversion of 4-bromopiperidine was complete. Furthermore, the deuterium incorporation level was estimated by an ESI-MS analysis. It was found that 0.9 deuterium atoms/piperidine molecules were incorporated on average, but isotopologous species containing two or three deuterium atoms were also identified (Figure 1). These results suggested that this precursor is satisfactory for achieving an approximate molar activity of $1 \mathrm{TBq} / \mathrm{mmol}$. In the next step, $\left[{ }^{3} \mathrm{H}\right] \mathrm{N}$-amino-piperidine was prepared from 4-bromopiperidine (Scheme 2). Reduction of the brominated piperidine with tritium gas in the presence of $\mathrm{Pd} / \mathrm{C}(10 \% \mathrm{Pd})$ catalyst resulted in tritium labelled piperidine that was nitrosated. The resulting $\left[{ }^{3} \mathrm{H}\right] \mathrm{N}$-nitrosopiperidine was then reduced with $\mathrm{LiAlH}_{4}$, and $\left[{ }^{3} \mathrm{H}\right] \mathrm{N}$-aminopiperidine was isolated as a hydrochloride salt. The carboxylic acid $\mathbf{5}$ was activated with $\mathrm{HOBt}$ in the presence of $\mathrm{DIC}$, and the resulting active ester was used in situ for the $\mathrm{N}$-acylation of $\left.{ }^{3} \mathrm{H}\right] \mathrm{N}$-aminopiperidine to give the tritium labelled anchor 8 . HPLC analysis revealed that a significant amount of tritium labelled $\mathrm{N}$-aminopiperidine was coupled to $\mathbf{5}$ (Figure 2). The purification of the crude cholesterol derivative was achieved by RP-HPLC, resulting in $17.4 \mathrm{MBq}$ of pure $\mathbf{8}$ with an isolated radiochemical yield of $17 \%$. The sterical hindrance of the carboxylic acid function of $\mathbf{5}$ and the more diluted reaction mixture as compared with that of $\mathbf{6}$ and $\mathbf{7}$ resulted in the low yield of $\mathbf{8}$. Quantitative analysis of the concentration and radioactivity of the labelled anchor $\mathbf{8}$ was performed by RP-HPLC via UV and radioactivity detection using a calibration curve made by 7 . The specific activity of the tritiated cholesterol anchor 8 was found to be $1.37 \mathrm{TBq} / \mathrm{mmol}$ that is higher than the theoretical value. Beyond tritium substitution of $\mathrm{Br}$ in 4-bromopiperidine, catalytic ${ }^{1} \mathrm{H} /{ }^{3} \mathrm{H}$ exchange reactions also took place presumably at positions 2 and 6 of the piperidine ring. When $2-3 \mathrm{~h}$ of reaction time is applied for catalytic dehalotritiation, the catalytic exchange becomes significant and increases the specific activity. ${ }^{23}$ The ESI-MS analysis of the deuterium labelled piperidine also indicated catalytic exchange reactions. It is important to note that the convergent synthetic strategy ensures that all the tritium atoms are localized in the piperidine ring of the headgroup, because after the tritium gas reduction of 4-bromopiperidine, further transformations do not affect the tritium distribution.

The control compound 6, a fluorescent GPI anchor mimetic, was used to assess the membrane association property of the designed $\left[{ }^{3} \mathrm{H}\right]$ anchor. The solubility of $\mathbf{6}$ was investigated in an aqueous medium before the imaging application. It was found that the amphiphile 6 partially dissolved in a serum-free medium as micellar associates and the remaining portion formed 

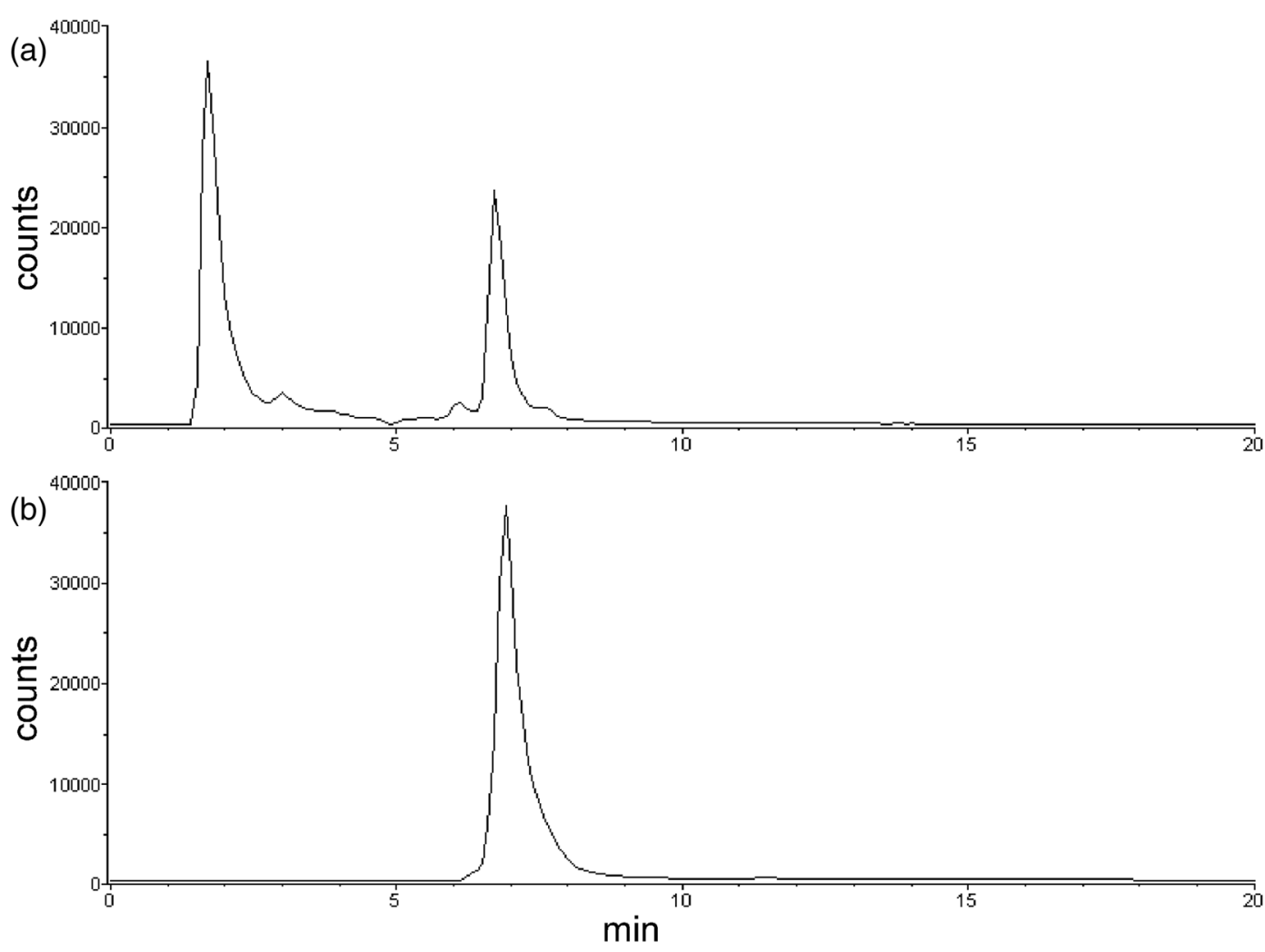

Figure 2. Radio-HPLC chromatogram of (a) the crude and (b) the purified tritium labelled cholesterol anchor $\left.8 .{ }^{3} \mathrm{H}\right] \mathrm{N}$-aminopiperidine was eluted without retention, and 8 was eluted at $t_{\mathrm{R}}=6.90 \mathrm{~min}$.

precipitates. The resulting heterogeneous mixture was incubated with 10 eq $\beta$-cyclodextrin that forms water-soluble inclusion complexes with cholesterol and $3 \beta$-hydroxyl-modified cholesterol derivatives. ${ }^{26}$ This way, $0.5 \mathrm{mM}$ aqueous solution of the cholesterol amphiphile was prepared. Then, SH-SY5Y cells were treated with $1 \mu \mathrm{M}$ of the inclusion complex of $\mathbf{6}$ for $30 \mathrm{~min}$. After delivering to live SH-SY5Y cells, an intensive membrane staining was observed, that is, $\mathbf{6}$ incorporated into the cell membrane similarly to our original anchor ${ }^{18}$ (Figure 3 ). It indicates that a slight modification in the $\alpha$-amino acid function of the headgroup is tolerated with the retention of the membrane association properties. The tritium labelled anchor $\mathbf{8}$ could also be solubilized in cell culture medium with this method, and it was applied for studying the cell membrane incorporation of $\mathbf{8}$. SH-SY5Y cells were incubated with 1-100 nM of $\mathbf{8}$ or $\mathbf{2}$ at $37^{\circ} \mathrm{C}$ for 10 and $30 \mathrm{~min}$, and then cells were washed and trypsinized. The membrane incorporated radioactivity was determined by liquid scintillation counting of the filtered cells. It was found that $\mathbf{2}$ was not incorporated into the cell membranes, as the radioactivity of these cell population was slightly above the background (Figure 4). However, when cells were treated with $\mathbf{8}$, concentration- and time-dependent incorporation of the radioactive cholesterol anchor was observed. The radioactivity of the cell cultures was ca. $20 \%$ higher after $30 \mathrm{~min}$ incubation than after $10 \mathrm{~min}$ incubation, indicating fast plasma membrane incorporation of $\mathbf{8}$.

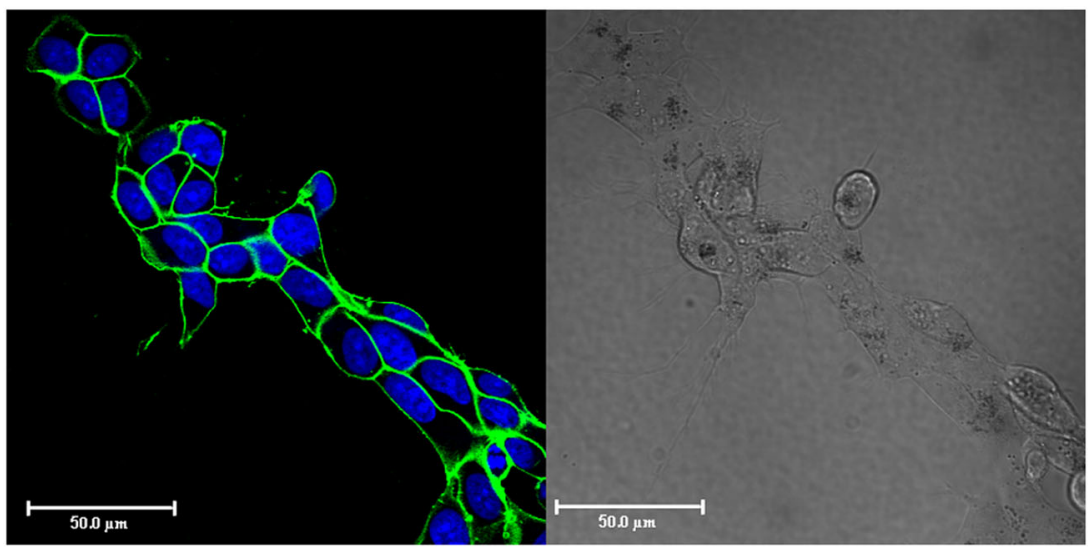

Figure 3. Confocal laser scanning (left) and differential interference contrast (right) microscopy of SH-SY5Y cells after 30 min incubation with $1 \mu \mathrm{M}$ cholesterol anchor 6 followed by a treatment with the nuclear dye DRAQ5 for $5 \mathrm{~min}$ at $37^{\circ} \mathrm{C}$. On the image, fluorescein is green and nuclei are blue. This figure is available in colour online at wileyonlinelibrary.com/journal/jlcr. 


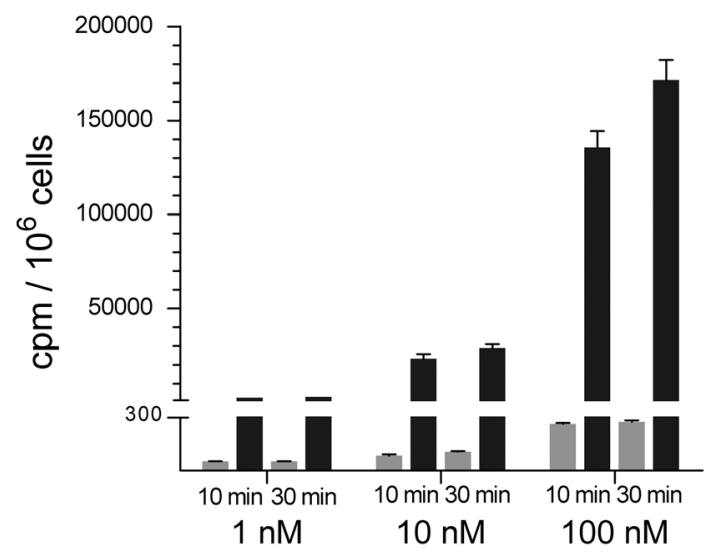

Figure 4. Incorporation of the $\left[{ }^{3} \mathrm{H}\right]$ cholesterol anchor 8 to the membrane of $\mathrm{SH}$ SY5Y cells. Cells were incubated with different concentrations of $\mathbf{2}$ (grey bars) or 8 (black bars) in the presence of $10 \mu \mathrm{M} \beta$-cyclodextrin followed by liquid scintillation counting of the washed and trypsinized cells. The error bars represent SEM values of three parallel measurements.

\section{Conclusions}

Semisynthetic lipoproteins prepared from recombinant proteins and designed lipid derivatives are valuable probes of the membrane-associated lipoproteins. In this article, we reported the synthesis of a novel tritium labelled cholesterol anchor that contains the radioactive label in the headgroup. It was achieved in a convergent synthetic approach by the acylation of $\left[{ }^{3} \mathrm{H}\right] \mathrm{N}$ aminopiperidine with the 1-hydroxybenzotriazole ester of the carboxylic acid derivative $\mathbf{5}$. This structure of the headgroup features the introduction of the tritium label into the same position where a fluorophore was introduced in $\mathbf{6}$. Thus, the fluorescein and tritium labelled anchors are constitutionally related, and the fluorescence microscopic images are relevant to the $\left[{ }^{3} \mathrm{H}\right]$ cholesterol incorporation data. Each synthetic step was optimized by using unlabelled standard compounds and by tracer experiments, and finally, the target cholesterol amphiphile $\mathbf{8}$ was obtained with a radiochemical purity of $98 \%$ and with a chemical purity of $99 \%$. It was demonstrated that the membrane incorporation of the resulting headgroup labelled $\left[{ }^{3} \mathrm{H}\right]$ cholesterol anchor is concentration and time dependent, and that it can be investigated at low concentrations, below the working concentration of fluorescent analogues. It is advantageous because under these conditions, the exogenous introduction of cholesterol derivative $\mathbf{8}$ results in low membrane concentration and thus low level of membrane perturbation, cellular stress.

\section{Acknowledgements}

Financial support from the Hungarian Scientific Research Fund (K77783), the Hungarian National Development Agency (TÁMOP
4.2.2.A-11/1/KONV-2012-0052) and the János Bolyai Research Scholarship of the Hungarian Academy of Sciences (Cs.T.), and technical help of Balázs Csibrány in cell culturing are acknowledged.

\section{Conflict of Interest}

The authors did not report any conflict of interest.

\section{References}

[1] T. Kinoshita, M. Fujita, chapter title: Overview of GPI biosynthesis. In The Enzymes. Volume 26: Glycosylphosphatidylinositol (GPI) Anchoring of Proteins (Eds.: A. K. Menon, T. Kinoshita, P. Orlean, F. Tamanoi), Academic Press: New York, 2009, pp. 1-30.

[2] M. E. Medof, T. Kinoshita, V. Nussenzweig, J. Exp. Med. 1984, 160, 1558.

[3] C. W. van den Berg, T. Cinek, M. B. Hallett, V. Horejsi, B. P. Morgan, J. Cell Biol. 1995, 131, 669.

[4] P. E. Milhiet, M. C. Giocondi, O. Baghdadi, F. Ronzon, B. Roux, C. Le Grimellec, EMBO Rep. 2002, 3, 485.

[5] C. Metzner, M. M. Mostegl, W. H. Gunzburg, B. Salmons, J. A. Dangerfield, FASEB J. 2008, 22, 2734.

[6] M. E. Medof, S. Nagarajan, M. L. Tykocinski, FASEB J. 1996, 10, 574.

[7] B. Bader, K. Kuhn, D. J. Owen, H. Waldmann, A. Wittinghofer, J. Kuhlmann, Nature 2000, 403, 223.

[8] D. R. Premkumar, Y. Fukuoka, D. Sevlever, E. Brunschwig, T. L. Rosenberry, M. L. Tykocinski, M. E. Medof, J. Cell. Biochem. 2001, 82, 234.

[9] F. R. Taylor, D. Wen, E. A. Garber, A. N. Carmillo, D. P. Baker, R. M. Arduini, K. P. Williams, P. H. Weinreb, P. Rayhorn, X. Hronowski, A. Whitty, E. S. Day, A. Boriack-Sjodin, R. I. Shapiro, A. Galdes, R. B. Pepinsky, Biochemistry 2001, 40, 4359.

[10] C. Peters, A. Wolf, M. Wagner, J. Kuhlmann, H. Waldmann, Proc. Natl. Acad. Sci. U. S. A. 2004, 101, 8531.

[11] M. J. Grogan, Y. Kaizuka, R. M. Conrad, J. T. Groves, C. R. Bertozzi, J. Am. Chem. Soc. 2005, 127, 14383.

[12] M. G. Paulick, M. B. Forstner, J. T. Groves, C. R. Bertozzi, Proc. Natl. Acad. Sci. U. S. A. 2007, 104, 20332.

[13] K. Teruya, K. Nishizawa, K. Doh-ura, Protein J. 2010, 29, 493.

[14] K. Simons, M. J. Gerl, Nat. Rev. Mol. Cell Biol. 2010, 11, 688.

[15] D. Lingwood, K. Simons, Science 2010, 327, 46.

[16] S. Agarwal, C. Schroeder, G. Schlechtingen, T. Braxmeier, G. Jennings, H. J. Knolker, Bioorg. Med. Chem. Lett. 2013, 23, 5165.

[17] S. B. Sato, K. Ishii, A. Makino, K. Iwabuchi, A. Yamaji-Hasegawa, Y. Senoh, I. Nagaoka, H. Sakuraba, T. Kobayashi, J. Biol. Chem. 2004, 279, 23790.

[18] B. Schäfer, E. Orbán, A. Borics, K. Huszár, A. Nyeste, E. Welker, Cs. Tömböly, Bioconj. Chem. 2013, 24, 1684.

[19] G. Tóth, S. Lovas, F. Ötvös, In Methods in Molecular Biology: Neuropeptide Protocols (Eds.: G. B. Irvine, C. H. Williams), Humana Press: Totowa, NJ, 1997, pp. 219-230.

[20] Cs. Tömböly, A. Péter, G. Tóth, Peptides 2002, 23, 1573.

[21] A. E. Christian, M. P. Haynes, M. C. Phillips, G. H. Rothblat, J. Lipid Res. 1997, 38, 2264.

[22] R. Leventis, J. R. Silvius, Biophys. J. 2001, 81, 2257.

[23] Cs. Tömböly, R. Dixit, I. Lengyel, A. Borsodi, G. Tóth, J. Label. Compd. Radiopharm. 2001, 44, 355.

[24] R. Hinman, J. Org. Chem. 1958, 23, 1587-1588.

[25] H. K. Hall Jr., J. Am. Chem. Soc. 1957, 79, 5441.

[26] R. Ravichandran, S. Divakar, J. Incl. Phenom. Mol. Recognit. Chem. 1998, 30, 253 